\title{
Article \\ Complex Modes of Inheritance in Hereditary Red Blood Cell Disorders: A Case Series Study of 155 Patients
}

\author{
Immacolata Andolfo ${ }^{1,2}$, Stefania Martone ${ }^{1,2}$, Barbara Eleni Rosato ${ }^{1,2}$, Roberta Marra ${ }^{1,2}$, Antonella Gambale ${ }^{2,3}$, \\ Gian Luca Forni ${ }^{4}$, Valeria Pinto ${ }^{4} \oplus$, Magnus Göransson ${ }^{5}$, Vasiliki Papadopoulou ${ }^{6}$, Mathilde Gavillet ${ }^{6}$, \\ Mohsen Elalfy ${ }^{7}\left(\mathbb{D}\right.$, Antonella Panarelli ${ }^{2}$, Giovanna Tomaiuolo ${ }^{2,8}$, Achille Iolascon ${ }^{1,2, *(\mathbb{C})}$ and Roberta Russo ${ }^{1}$ (])
}

1 Dipartimento di Medicina Molecolare e Biotecnologie Mediche, Università degli Studi di Napoli Federico II, 80131 Napoli, Italy; andolfo@ceinge.unina.it (I.A.); stefaniamartone85@libero.it (S.M.); rosato.barbara@gmail.com (B.E.R.); robertamarra.r@gmail.com (R.M.); roberta.russo@unina.it (R.R.)

2 CEINGE Biotecnologie Avanzate, 80145 Naples, Italy; antonellagambale@gmail.com (A.G.); panarelli@ceinge.unina.it (A.P.); g.tomaiuolo@unina.it (G.T.)

3 Department of Laboratory Medicine (DAIMedLab), UOC Medical Genetics, 'Federico II' University Hospital, 80131 Naples, Italy

4 Centro della Microcitemia e delle Anemie Congenite, Ospedale Galliera, 16128 Genoa, Italy; gianluca.forni@galliera.it (G.L.F.); valeria.pinto@galliera.it (V.P.)

5 Department of Paediatrics, The Queen Silvia Children's Hospital, Sahlgrenska University Hospital, 41345 Gothenburg, Sweden; magnus.l.goransson@vgregion.se

6 Service and Central Laboratory of Haematology, Department of Oncology and Department of Laboratory Medicine and Pathology, Lausanne University Hospital (CHUV), 1011 Lausanne, Switzerland; vasiliki.papadopoulou@chuv.ch (V.P.); mathilde.gavillet@chuv.ch (M.G.)

check for updates

Citation: Andolfo, I.; Martone, S.; Rosato, B.E.; Marra, R.; Gambale, A.; Forni, G.L.; Pinto, V.; Göransson, M.; Papadopoulou, V.; Gavillet, M.; et al. Complex Modes of Inheritance in Hereditary Red Blood Cell Disorders: A Case Series Study of 155 Patients. Genes 2021, 12, 958. https://doi.org/ $10.3390 /$ genes 12070958

Academic Editor: Julia Horsfield

Received: 27 May 2021

Accepted: 19 June 2021

Published: 23 June 2021

Publisher's Note: MDPI stays neutral with regard to jurisdictional claims in published maps and institutional affiliations.

Copyright: (c) 2021 by the authors. Licensee MDPI, Basel, Switzerland. This article is an open access article distributed under the terms and conditions of the Creative Commons Attribution (CC BY) license (https:/ / creativecommons.org/licenses/by/ $4.0 /)$.
7 Thalassemia Centre, Faculty of Medicine, Ain Shams University, Cairo 11566, Egypt; elalfym@hotmail.com

8 Department of Chemical Engineering, Materials and Industrial Production, 'Federico II' University of Naples, 80125 Naples, Italy

* Correspondence: achille.iolascon@unina.it

\begin{abstract}
Hereditary erythrocytes disorders include a large group of conditions with heterogeneous molecular bases and phenotypes. We analyzed here a case series of 155 consecutive patients with clinical suspicion of hereditary erythrocyte defects referred to the Medical Genetics Unit from 2018 to 2020. All of the cases followed a diagnostic workflow based on a targeted next-generation sequencing panel of 86 genes causative of hereditary red blood cell defects. We obtained an overall diagnostic yield of $84 \%$ of the tested patients. Monogenic inheritance was seen for $69 \%(107 / 155)$, and multi-locus inheritance for 15\% (23/155). PIEZO1 and SPTA1 were the most mutated loci. Accordingly, 16/23 patients with multi-locus inheritance showed dual molecular diagnosis of dehydrated hereditary stomatocytosis/xerocytosis and hereditary spherocytosis. These dual inheritance cases were fully characterized and were clinically indistinguishable from patients with hereditary spherocytosis. Additionally, their ektacytometry curves highlighted alterations of dual inheritance patients compared to both dehydrated hereditary stomatocytosis and hereditary spherocytosis. Our findings expand the genotypic spectrum of red blood cell disorders and indicate that multi-locus inheritance should be considered for analysis and counseling of these patients. Of note, the genetic testing was crucial for diagnosis of patients with a complex mode of inheritance.
\end{abstract}

Keywords: red blood cell defects; targeted next-generation sequencing; multi-locus inheritance; PIEZO1; SPTA1

\section{Introduction}

Hereditary anemias are a heterogeneous group of conditions that are characterized by complex genotype-phenotype correlations. Based on clinical manifestations and morphological red blood cell (RBC) alterations, hereditary anemias can be broadly classified into four different subtypes: (i) disorders of hemoglobin synthesis, such as thalassemia 
and hemoglobinopathies; (ii) hypo-regenerative anemias, such as congenital dyserythropoietic anemias; (iii) aregenerative anemias, such as Diamond-Blackfan anemia (iv) RBC membrane defects that are due to either alterations to the structural organization of the membranes, such as hereditary spherocytosis (HS), hereditary pyropoikilocytosis (HPP), and hereditary elliptocytosis, or to alterations to membrane transport functions, such as hereditary stomatocytosis; and (v) nonspherocytic hemolytic anemias, due to RBC enzyme defects [1-7].

The conventional workflow for diagnosis of hereditary anemias starts with the first line of investigation of evaluation of familial history, with complete blood counts and peripheral blood smears. Then, specialized biochemical tests are required. Finally, genetic testing serves as the confirmatory test. Currently, genetic testing is used early in the diagnostic workflow of hereditary anemias, which removes the need for some of the specialized tests [8,9], especially when the clinical data for the patients are not informative, or when the patient is transfusion dependent. Next-generation sequencing (NGS), as mainly the targeted NGS approach, has revolutionized the framework of the diagnosis of hereditary anemias by reducing both time and cost. Nevertheless, it remains a challenge to diagnose many hereditary anemia phenotypes according to phenotypic features and conventional diagnostic testing. In general clinical genetics setting, the diagnostic yield ranges from $38 \%$ to $87 \%$ of patients, depending on how many and which genes are included, and on the depth of the phenotypic assessment required [9]. A drawback of NGS-based genetic testing remains the data analysis, which includes several variants of unknown significance. Functional tests are therefore crucial to assess the pathogenicity of new variants that are detected by NGS.

One of the major advantages of the NGS approach is the identification of both polygenic conditions and modifier variants associated with causative mutations. Indeed, studies of Mendelian conditions have revealed the extent to which many rare diseases can be characterized by complex modes of inheritance, such as digenic inheritance and dual molecular diagnoses, which occur when pathogenic variations at two or more loci lead to expression of two or more Mendelian conditions [10].

In this study, we evaluated a large case series of 155 consecutive patients with different forms of hereditary anemias and erythrocytosis who were referred to the Medical Genetics Unit ('Federico II' University Hospital, Naples, Italy) for NGS-based genetic testing, from January 2018 to September 2020. Among the diagnosed patients, 15\% showed multi-locus inheritance, which mainly involved PIEZO1 and SPTA1 variants.

\section{Materials and Methods}

\subsection{Patients and Genomic DNA Preparation}

In total, 155 patients with clinical suspicion of different types of hereditary anemia were included in this study. Their diagnoses were based on history, clinical findings, and laboratory data. For HS/hereditary stomatocytosis patients, the diagnosis was also based on ektacytometry.

The local University Ethical Committee approved the collection of the patient data (DAIMedLab, 'Federico II' University of Naples; $N^{\circ}$ 252/18). DNA samples were obtained from the patients after they had signed their informed consent, and according to the Declaration of Helsinki. Whenever possible, affected and unaffected relatives were also enrolled to correctly assess the pathogenicity of each variant by analysis of the family segregation.

Genomic DNA preparation was performed as previously described [11]. To evaluate the quality of the extracted genomic DNA before fragmentation, samples were quantified using a UV-Vis spectrophotometer (NanoDrop 2000; Thermo Scientific, Waltham, MA, USA). Then, the genomic DNA was run on $0.8 \%$ agarose DNA gel electrophoresis.

\subsection{Libraries Establishment}

Genetic testing was achieved by targeted NGS using a custom 86-gene panel for hereditary RBC defects [12]. This panel is an updated version of a similar previously published panel [13], and it was composed of 86 genes that are causative of congenital dyserythropoietic anemias, Diamond-Blackfan anemia, RBC membrane defects, hemolytic 
anemias due to RBC enzyme defects, anemias due to iron metabolism defects, hereditary hemochromatosis, and hereditary erythrocytosis.

For the probe design, coding regions, $5^{\prime} \mathrm{UTR}, 3^{\prime} \mathrm{UTR}$, and 50-bp flanking splice junctions were selected as the regions of interest. The probe design was performed using the web-based tool SureDesign (https: / / earray.chem.agilent.com/suredesign.htm, accessed on 2 August 2019; Agilent Technologies, Santa Clara, CA, USA). The sequence length was set at $150 \times 2$ nucleotides. The total probe size was $298,393 \mathrm{kbp}$. Sample preparation was performed using target enrichment (SureSelectQXT) for the Illumina platform (SureSelect Custom Tier1 1-499 kb; Agilent Technologies, Santa Clara, CA, USA), according to the manufacturer's instructions.

\subsection{Sequencing and Data Analysis}

High-throughput sequencing was performed using a benchtop sequencer (MiSeq; Illumina). The alignment of sequencing reads to the genomic locations, quality control metrics, and identification of variants were achieved using the Alissa Align and Call software (v1.1.2-2; Agilent Technologies, Santa Clara, CA, USA). Variant annotation and analysis were performed using the Alissa Interpret software (v5.2.6; Agilent Technologies, Santa Clara, CA, USA). As previously described and according to the guidelines of the American College of Medical Genetics and Genomics (ACMG), the pathogenicity of each variant was evaluated by gathering evidence from various sources: population data, computational and predictive data, functional data, and segregation data [13].

Due to the large range of prevalence in the population of these heterogeneous disorders, we selected both rare and low-frequency variants (minor allele frequency: $<0.01$, 0.05 , respectively), as reported by the gnomAD browser (https:/ / gnomad.broadinstitute. org/ accessed on 5 April 2021). The InterVar (http:/ / wintervar.wglab.org/ accessed on 5 April 2021) and Varsome (https:/ / varsome.com/ accessed on 5 April 2021) web tools were used for clinical interpretation of the new variants, following the ACMG and the Association for Molecular Pathology guidelines [14]. Automated output was adjusted using the available evidence for each patient (Table 1 and Table S1). For the functional data criteria for the new variants, we did not perform in vitro functional studies that are supportive of a damaging effect on the gene or gene product, as needed to obtain strong evidence of pathogenicity. However, we selected and report those variants with moderate pathogenic evidence, i.e., variants located in a mutational hot spot and/or critical and well-established functional domain. Moreover, the validation of the variants was assessed by analysis of clinical data and family history, ektacytometry analysis, and peripheral blood evaluation, whenever possible.

All of the prioritized variants were confirmed by Sanger sequencing and by analysis of inheritance patterns, whenever possible. The validations were performed using 50 ng genomic DNA. Custom primers were designed using the Primer3 software (v. 0.4.0; freeware online). The primer sequences are available on request (roberta.russo@unina.it). Nucleotide numbering reflects cDNA numbering with +1 corresponding to the ' $\mathrm{A}$ ' of the ATG translation initiation codon in the reference sequence, according to the nomenclature for the description of sequence variants of the Human Genome Variation Society (www. hgvs.org/mutnomen, accessed on 5 April 2021). The initiation codon is codon 1.

\subsection{Gene Ranking}

To rank the mutated genes based on their genic intolerance, we used the Residual Variation Intolerance Score (RVIS) percentile, as retrieved from the Genic Intolerance database (http:/ / genic-intolerance.org/, accessed on 5 April 2021). The RVIS is a genebased score that is designed to rank genes in terms of whether they have more or less common functional genetic variation relative to the genome-wide expectation, given the amount of apparently neutral variation the gene has. A gene with a positive score has more common functional variation, and a gene with a negative score has less, and is referred to as 'intolerant' [15]. 
Table 1. Variant classification and reassessment.

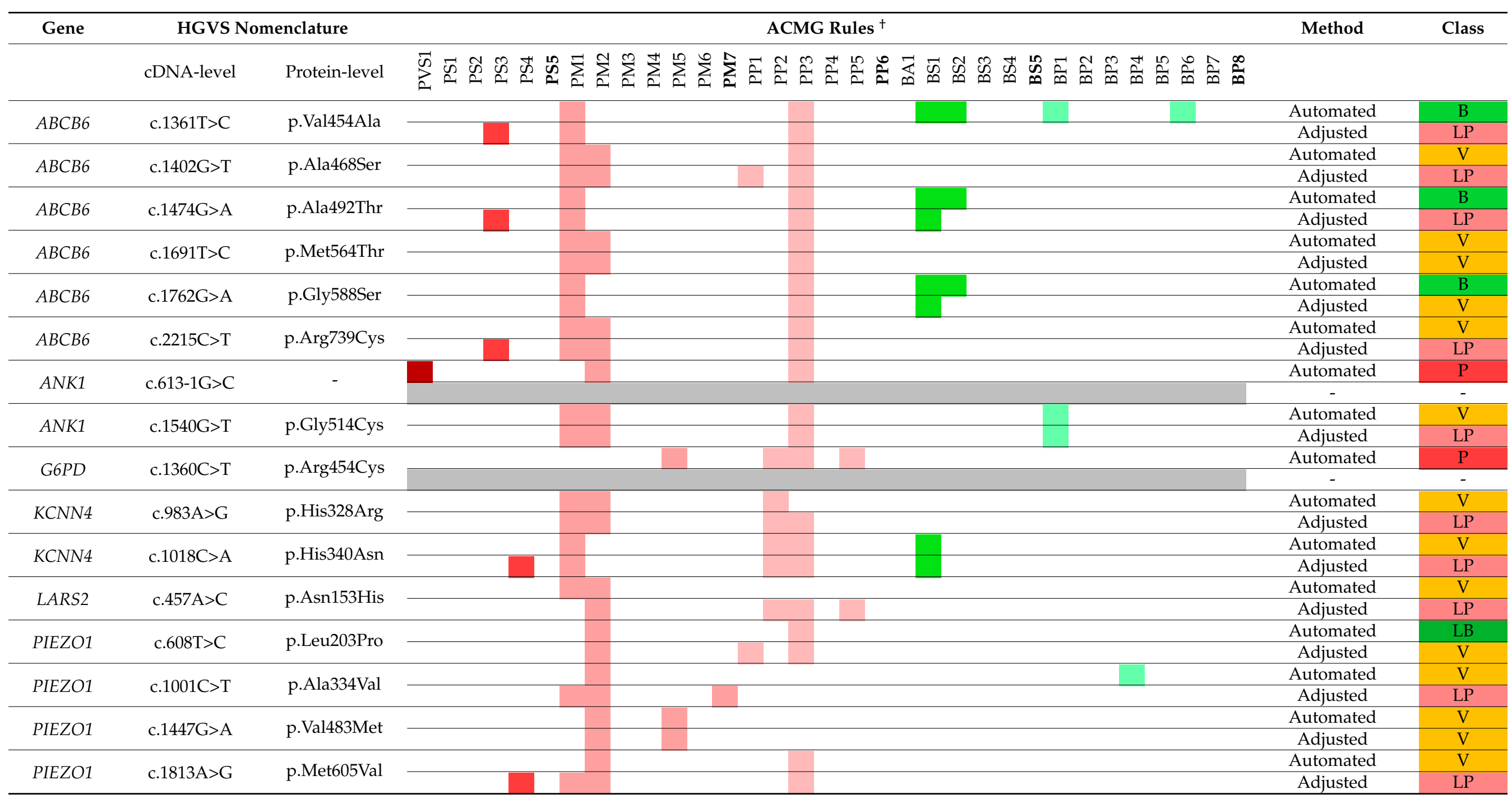


Table 1. Cont.

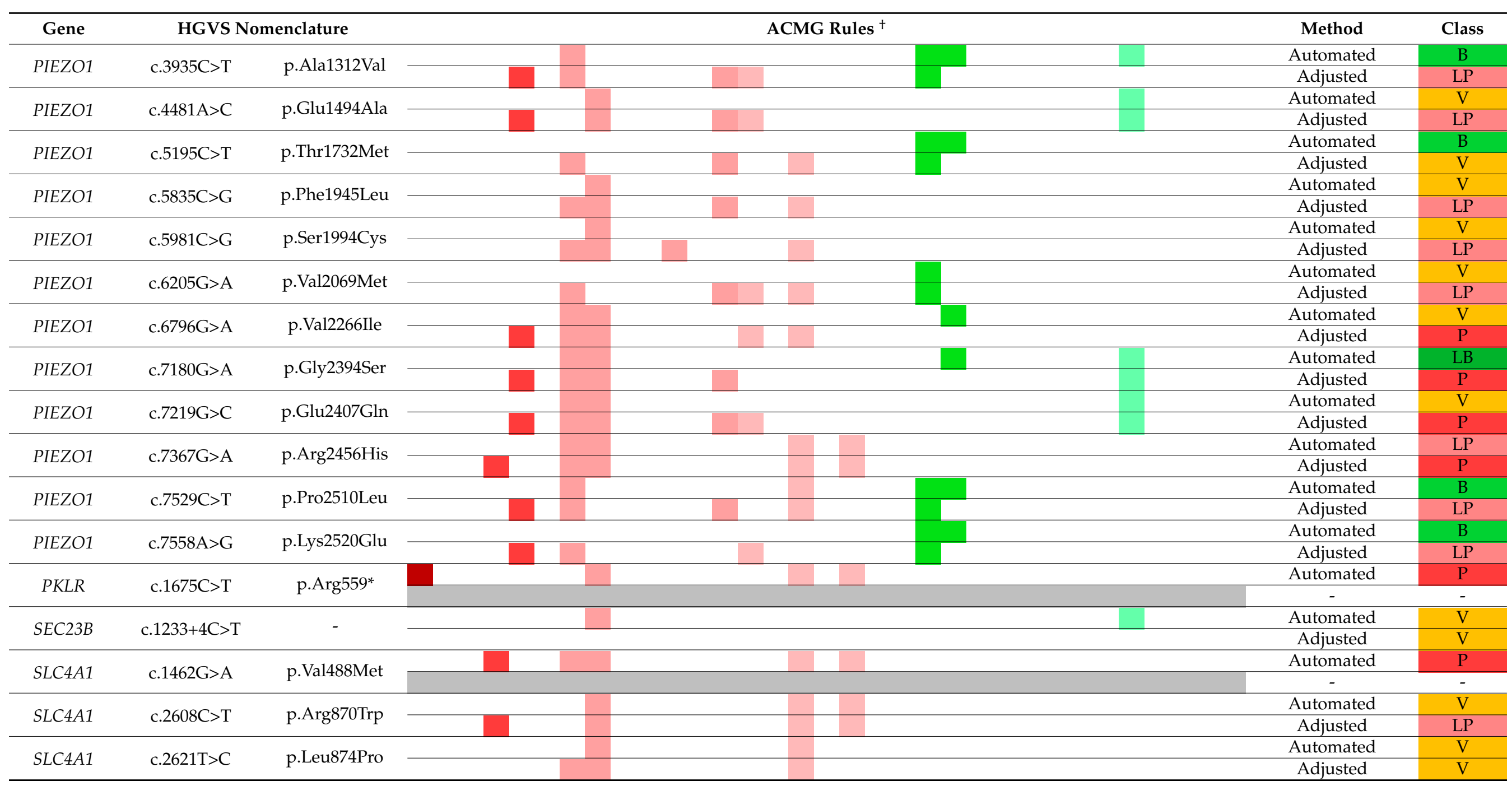


Table 1. Cont.

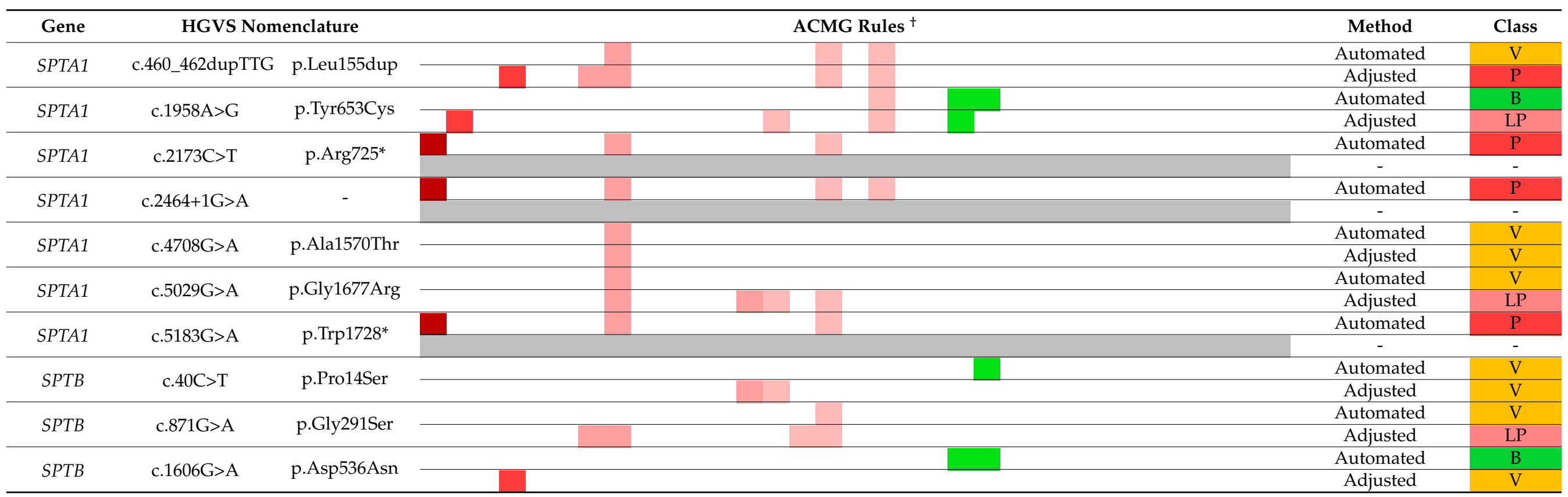

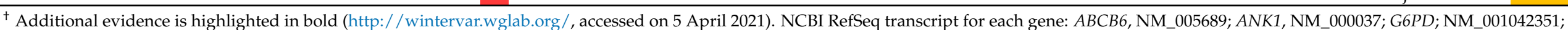

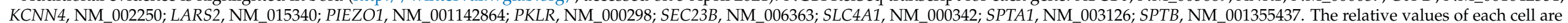

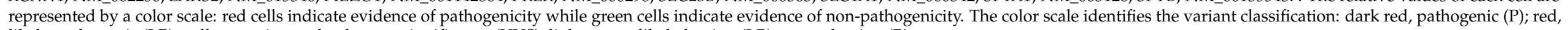
likely pathogenic (LP); yellow, variants of unknown significance (VUS); light green, likely benign (LB); green, benign (B). 


\subsection{Statistical Analysis}

Quantitative data were compared using Mann-Whitney tests. Multiple comparisons were performed using Kruskal-Wallis tests, with post-hoc correction using Dunn's multiple comparison tests. Qualitative data were compared using chi-squared tests. A two-sided $p<0.05$ was considered as statistically significant.

\section{Results}

\subsection{NGS-Based Genetic Testing for Identification of Multiple Disease-Causing Genotypes}

Among the 155 patients originally suspected of red blood cell defects, final diagnoses were reached for 130/155 (84\%). Overall, 69\% (107/155) showed monogenic inheritance, and $15 \%(23 / 155)$ showed multi-locus inheritance (Figure 1A). The genetic features of the patients with multi-locus inheritance patterns are summarized in Table 2. The complete clinical features were available for only 20/23 patients within this subset (Table S2).

A

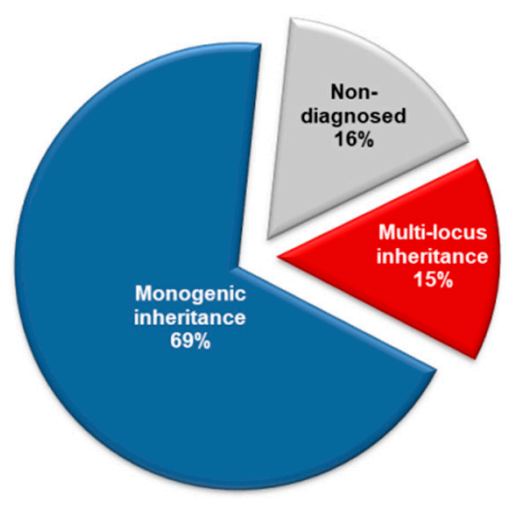

B

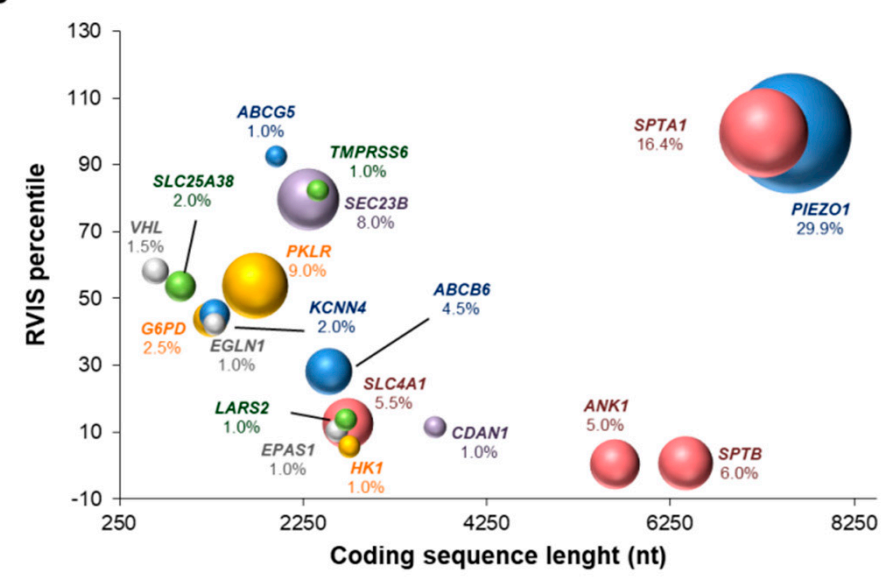

Figure 1. Molecular features of patients with hereditary red blood cell disorders. (A) Pie chart showing the proportions of patients diagnosed as monogenic (single gene condition) and multi-locus diseases. The undiagnosed cases evaluated by extended targeted next-generation sequencing for hereditary anemias are also shown. (B) Bubble chart defining the lengths of the coding sequences of each hereditary anemia causative gene and their relative Residual Variation Intolerance Score (RVIS) percentiles. Low RVIS percentiles identify increased constraints (intolerance to variation). The size of each bubble represents the frequency of the mutations in each gene, as calculated by the ratio of the number of mutated alleles for each gene and the overall count of disease alleles $(n=207)$.

Among the patients with multi-locus molecular diagnosis, 18/23 (78\%) showed variants of the PIEZO1 gene, and 7/23 (30\%) showed SPTA1 variants. In agreement with this, PIEZO1 and SPTA1 were the most mutated loci among the other causative genes identified in this case series (Figure 1B). Of note, the high frequencies of mutations in both of these genes were mainly related to their low genic intolerance, as suggested by the high values of the RVIS percentiles for both genes (Figure 1B). Accordingly, most of the variants in PIEZO1 and SPTA1 genes were originally predicted as variants of uncertain significance (VUS) or likely benign (Table 1). Interestingly, the reevaluation of PIEZO1 and SPTA1 pathogenic variants by ACMG rules demonstrated that 26/35 (74\%) and 17/35 (48.6\%) PIEZO1 variants were predicted as VUS by InterVar and Varsome tools, respectively. Similarly, SPTA1 pathogenic variants were predicted as VUS in a range from $43.8 \%$ (Varsome) to $93.8 \%$ (InterVar) (Figure S1). Accordingly, whenever possible, automated outputs were adjusted and explained using the available evidence for each patient (Table 1 and Table S1).

\subsection{Blood Count, Hemolytic Markers, and Iron Balance of Dual Inheritance Patients}

In total, 16 of the 23 patients with multi-locus diagnosis (70\%) showed dual molecular diagnosis of hereditary stomatocytosis, most of which were affected by dehydrated hereditary stomatocytosis type 1 (DHS1), and hereditary spherocytosis mainly due to 
biallelic SPTA1 variants (Table 2). Here, we named this combination as "dual inheritance". The family segregation of the variants identified in some of the patients with multi-locus inheritance is summarized in Figure S2.

To investigate the multi-locus contributions to the hematological phenotype in these dual inheritance patients (only those patients with dual inheritance of DHS and HS ( $n=16$ ) with code P1, P3-P9, P11-P15, P18_P20, indicated in bold in Table 2), some RBC indices were compared with those with DHS1 $(n=37$, patients with clinical and molecular diagnosis described in [16]) and HS $(n=21$, patients with clinical and molecular diagnosis included in the 155 ones here described): hemoglobin $(\mathrm{Hb})$, mean corpuscular volume [MCV], mean corpuscular hemoglobin [MCH], and ferritin:age ratio (Figure 2A and Table 3). Overall, there were no differences in these RBC indices between dual inheritance and HS, while significant differences were seen for dual inheritance versus DHS1 (Figure 2A and Table 3). Indeed, dual inheritance patients showed lower $\mathrm{Hb}, \mathrm{MCV}$, and $\mathrm{MCH}$ compared to DHS1 patients. As expected, MCV and MCH were higher in DHS1 patients than HS patients (Figure 2A and Table 3). Interestingly, DHS1 patients showed significantly higher ferritin:age ratio compared to both dual inheritance and HS patients (Figure 2A and Table 3).

A
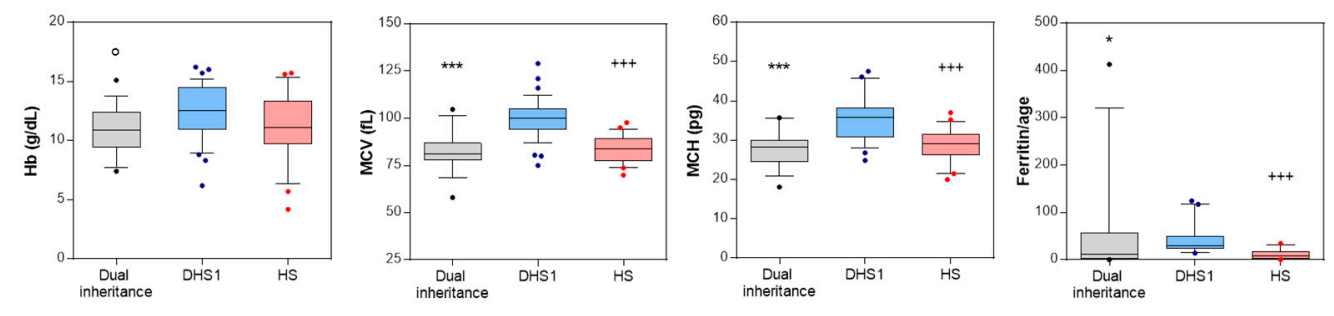

B
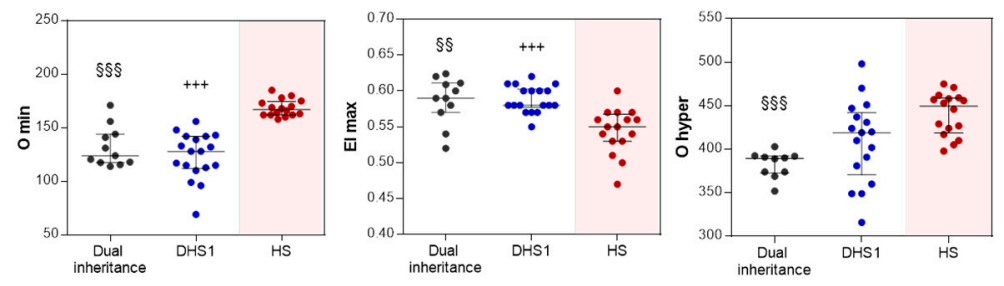

Figure 2. Hematological features and Osmoscan indices of digenic/oligogenic patients. (A) Hemoglobin $(\mathrm{Hb})(n=16)$, mean corpuscular volume $(\mathrm{MCV})(n=16)$, mean corpuscular hemoglobin $(\mathrm{MCH})(n=16)$, and ferritin/age levels in patients with dual inheritance $(n=16$, patients indicated in bold in Table 2), dehydrated hereditary stomatocytosis type 1 (DHS1; $n=37$ ), and hereditary spherocytosis (HS; $n=21$ ). Data are medians and whiskers for 10-90 percentiles. (B). O min, EI max, and O hyper values from Osmoscan profiles of patients with dual inheritance $(n=11)$, DHS1 $(n=18)$, and HS $(n=16)$. Data are medians and interquartile range. ${ }^{\circ}, p<0.05$ (dual inheritance vs. DHS1, Student's $t$ test). ${ }^{*}, p<0.05 ;{ }^{* * *}, p<0.0001$ (dual inheritance vs. DHS1). ,$+++ p<0.0001$ (HS vs. DHS1). $\S \S, p<0.001$ (dual inheritance vs. HS). $\S \S \S, p<0.0001$ (dual inheritance vs. HS) (Kruskal-Wallis tests, with post-hoc correction for internal comparisons by Dunn's multiple comparison tests). 
Table 2. Genetic features of the patients with multi-locus inheritance.

\begin{tabular}{|c|c|c|c|c|c|c|c|c|}
\hline \multirow{2}{*}{ Patient ID } & \multirow{2}{*}{ Disease } & \multirow{2}{*}{ Gene } & \multicolumn{2}{|c|}{ HGVS Nomenclature } & \multirow{2}{*}{ Zygosity } & \multirow{2}{*}{ RefSeq ID } & \multirow{2}{*}{$\begin{array}{c}\mathrm{AF} \\
\text { gnom } \mathrm{AD} \S\end{array}$} & \multirow[t]{2}{*}{ HGMD ID } \\
\hline & & & cDNA-Level & Protein-Level & & & & \\
\hline \multirow[t]{3}{*}{ P1 } & HS/FP & $A B C B 6$ & NM_005689:c.C2215T & p.Arg739Cys & Het & rs141840760 & 0.0004 & - \\
\hline & & SPTA1 & NM_003126:c.5183G>A & p.Trp $1728^{*}$ & Comp het & - & - & - \\
\hline & & SPTA1 & NM_003126:c.6531-12C>T & $\begin{array}{c}- \\
t\end{array}$ & Comp het & rs28525570 & 0.23 & CS995155 \\
\hline \multirow[t]{3}{*}{$\mathrm{P} 2$} & HS/CDAII & SEC23B & NM_006363:c.1233+4C>T & - & Hom & rs201883785 & - & - \\
\hline & & SPTA1 & NM_003126:c.4708G>A & p.Ala1570Thr & Hom & rs778626016 & - & - \\
\hline & & SPTA1 & NM_003126:c.6531-12C>T & - & Hom & rs 28525570 & 0.23 & CS995155 \\
\hline \multirow{2}{*}{ P3 } & & SPTA1 & NM_003126:c.5029G>A & p.Gly1677Arg & Comp het & rs771033064 & 0 & CM187374 \\
\hline & & PIEZO1 & NM_001142864:c.7558A>G & p.Lys2520Glu & Het & rs570744198 & 0.001 & CM187408 \\
\hline \multirow[t]{2}{*}{$\mathbf{P 4}$} & DHS1/HE & SPTB & NM_001355437:c.871G>A & p.Gly291Ser & Het & rs143599352 & 0.0002 & - \\
\hline & & PIEZO1 & NM_001142864:c.7219G >C & p.Glu2407Gln & Het & rs200291894 & 0.0001 & CM1922287 \\
\hline \multirow[t]{2}{*}{ P5 } & DHS1/HE & SPTB & NM_001355437:c.40C>T & p.Pro14Ser & Het & rs147059670 & 0.0001 & - \\
\hline & & PIEZO1 & NM_001142864:c.7180G>A & p.Gly2394Ser & Het & rs201950081 & 0.0001 & CM187364 \\
\hline P6 & DHS1/HS & PIEZO1 & NM_001142864:c.608T>C & p.Leu203Pro & Het & rs977249154 & 0 & - \\
\hline \multirow[t]{2}{*}{ P7 } & DHS1/HS & PIEZO1 & NM_001142864:c.4481A>C & p.Glu1494Ala & Het & - & - & - \\
\hline & & SPTA1 & NM_003126:c.2464+1G>A & - & Hom & rs774632615 & 0 & - \\
\hline \multirow[t]{3}{*}{ P8 } & DHS2/FP/HLASA & $A B C B 6$ & NM_005689:c.1402G>T & p.Ala468Ser & Het & rs777270402 & 0 & - \\
\hline & & LARS2 & NM_015340:c.457A>C & p.Asn $153 \mathrm{His}$ & Hom & rs786205560 & - & CM1615275 \\
\hline & & KCNN4 & NM_002250:c.1018C>A & p.His340Asn & Het & rs76935412 & 0.002 & - \\
\hline \multirow[t]{2}{*}{ P9 } & HS/FP & $A B C B 6$ & NM_005689:c.1762G>A & p.Gly588Ser & Het & rs145526996 & 0.004 & CM128905 \\
\hline & & SLC4A1 & NM_000342:c.2621T>C & p.Leu874Pro & Het & - & - & - \\
\hline \multirow[t]{2}{*}{ P10 } & PKD/DHS1 & PKLR & NM_000298:c.1675C>T & p.Arg559* & Hom & rs532230312 & 0 & CM981585 \\
\hline & & PIEZO1 & NM_001142864:c.6796G>A & p.Val2266Ile & Het & rs546338962 & 0 & CM187363 \\
\hline \multirow[t]{2}{*}{ P11 } & DHS1/HE & PIEZO1 & NM_001142864:c.5195C>T & p.Thr1732Met & Het & rs139051768 & 0.011 & - \\
\hline & & SPTB & NM_001024858:c.1606G>A & p.Asp536Asn & Het & rs145675502 & 0.001 & CM187385 \\
\hline \multirow[t]{2}{*}{ P12 } & DHS1/FP & PIEZO1 & NM_001142864:c.7367G>A & p.Arg2456His & Het & rs587776988 & - & CM127746 \\
\hline & & $A B C B 6$ & NM_005689:c.1474G>A & p.Ala492Thr & Het & rs147445258 & 0.007 & CM169662 \\
\hline
\end{tabular}


Table 2. Cont.

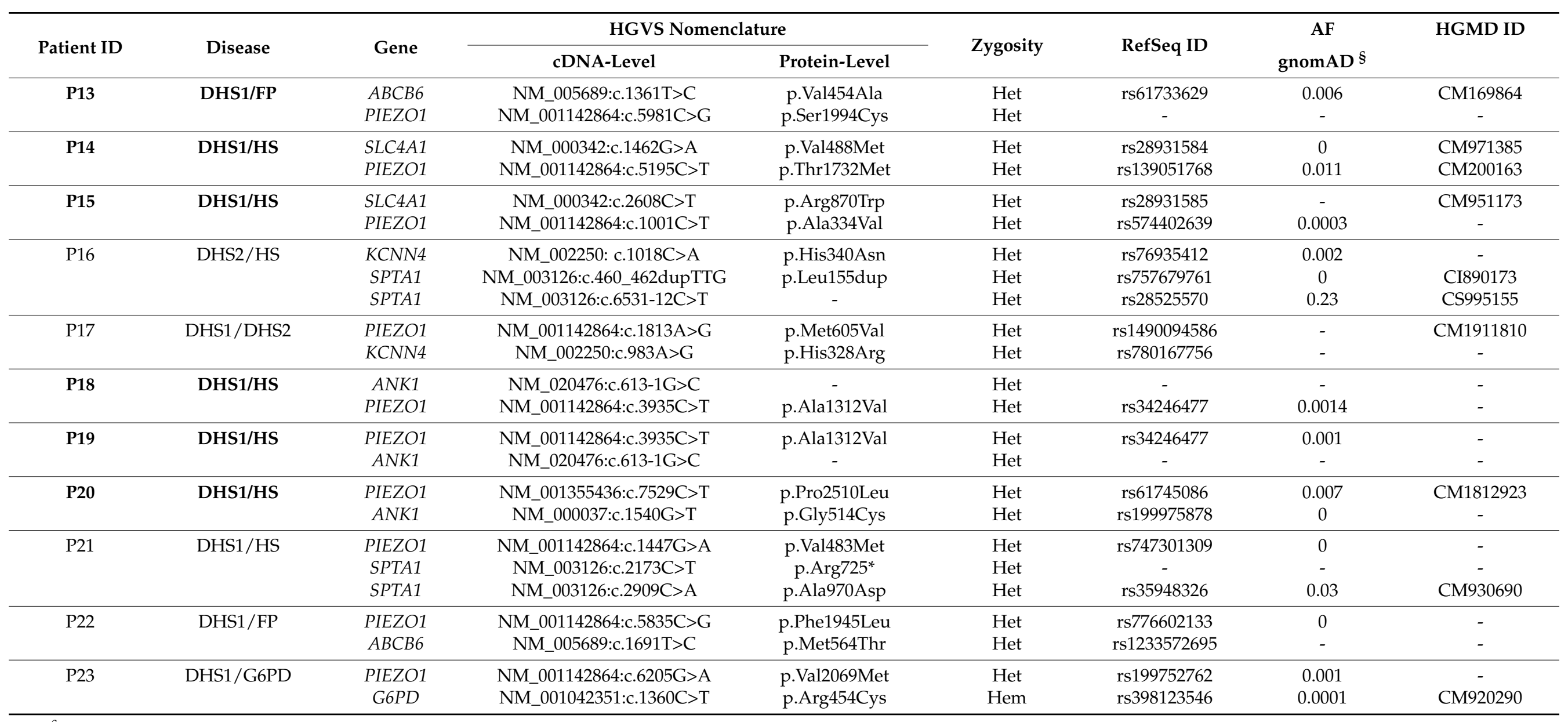

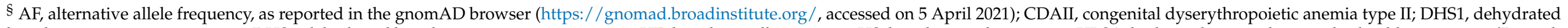

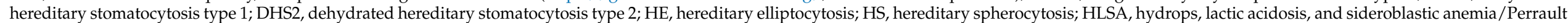

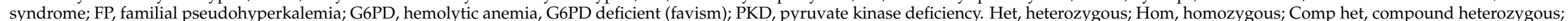
HGVS, Human Genome Variation Society database; HGMD, Human Gene Mutation database (HGMD Professional 2020.3). In bold are indicated the patients analyzed in Figure 2. 
Table 3. Clinical features of the case series enrolled in the study.

\begin{tabular}{|c|c|c|c|c|c|c|c|c|c|}
\hline Analysis & Unit & $\begin{array}{l}\text { DHS1 Cases } \\
(n=37)\end{array}$ & $\begin{array}{l}\text { HS Cases } \\
(n=21)\end{array}$ & $\begin{array}{l}\text { Dual Inheritance } \\
(n=16)\end{array}$ & Reference Range & $\mathbf{P} \S$ & $\mathbf{P}^{1}$ & $\mathbf{P}^{2}$ & $\mathbf{P}^{3}$ \\
\hline Age & years & $20.5 \pm 3.2(20.0 ; 28)$ & $27.8 \pm 4.0(25.0 ; 20)$ & $23.9 \pm 5.1(18.5 ; 16)$ & - & 0.32 & - & - & - \\
\hline Gender & female/male & $17(45.9) / 20(54.1)$ & $16(76.2) / 5(23.8)$ & $5(29.4) / 11(70.6)$ & - & 0.02 & 0.32 & $<0.01$ & 0.03 \\
\hline \multicolumn{10}{|l|}{ Hematological data } \\
\hline RBCs & $\times 10^{6} / \mu \mathrm{L}$ & $3.1 \pm 0.1(3.0 ; 36)$ & $3.9 \pm 0.2(4.3 ; 19)$ & $4.0 \pm 0.2(3.9 ; 15)$ & $4.0-5.2$ & $<0.001$ & 0.004 & 1.00 & 0.004 \\
\hline $\mathrm{Hb}$ & $\mathrm{g} / \mathrm{dL}$ & $12.5 \pm 0.4(12.7 ; 36)$ & $11.2 \pm 0.6(11.1 ; 21)$ & $10.9 \pm 0.5(10.8 ; 16)$ & $11.5-15.5$ & 0.06 & - & - & - \\
\hline $\mathrm{Ht}$ & $\%$ & $36.3 \pm 1.7(37.3 ; 36)$ & $33.1 \pm 1.6(33.1 ; 19)$ & $31.8 \pm 1.6(30.5 ; 14)$ & $35-45$ & 0.04 & 0.11 & 1.00 & 0.14 \\
\hline $\mathrm{MCV}$ & $\mathrm{fL}$ & $100.5 \pm 1.7(100.0 ; 36)$ & $83.2 \pm 1.6(83.8 ; 21)$ & $82.5 \pm 2.5(80.9 ; 16)$ & 77-95 & $<0.001$ & $<0.001$ & 1.00 & $<0.001$ \\
\hline $\mathrm{MCH}$ & pg & $35.9 \pm 1.1(36.0 ; 27)$ & $28.8 \pm 0.9(29.0 ; 21)$ & $27.6 \pm 1.1(27.4 ; 16)$ & $25-33$ & $<0.001$ & $<0.001$ & 1.00 & $<0.001$ \\
\hline $\mathrm{MCHC}$ & $\mathrm{g} / \mathrm{dL}$ & $35.5 \pm 0.7(34.3 ; 36)$ & $34.2 \pm 0.9(34.5 ; 19)$ & $34.1 \pm 0.5(34.1 ; 16)$ & $32-36$ & 0.81 & - & - & - \\
\hline PLTs & $\times 10^{3} / \mu \mathrm{L}$ & $414.2 \pm 55.3(375.0 ; 15)$ & $276.3 \pm 34.1(270.0 ; 20)$ & $288.7 \pm 41.8(232.0 ; 16)$ & $150-450$ & 0.05 & 0.08 & 1.00 & 0.09 \\
\hline ARC & $\times 10^{3} / \mu \mathrm{L}$ & $164.5 \pm 17.9(145.9 ; 33)$ & $238.3 \pm 45.9(234.6 ; 16)$ & $217.5 \pm 50.5(144.5 ; 13)$ & $20-90$ & 0.49 & - & - & - \\
\hline \multicolumn{10}{|c|}{ Biochemical data and iron balance } \\
\hline Total bilirubin & $\mathrm{mg} / \mathrm{dL}$ & $3.7 \pm 0.6(3.9 ; 21)$ & $2.9 \pm 0.6(2.4 ; 16)$ & $3.0 \pm 0.5(3.5 ; 12)$ & $0.3-1.0$ & 0.59 & - & - & - \\
\hline Ferritin & $\mathrm{ng} / \mathrm{mL}$ & $491.3 \pm 82.1(363.0 ; 19)$ & $319.1 \pm 117.8(150.2 ; 14)$ & $409.8 \pm 154.4(128.5 ; 13)$ & $22-275$ & 0.18 & - & - & - \\
\hline Ferritin level/dosage age $^{+}$ & - & $38.9 \pm 6.5(30.3 ; 18)$ & $10.9 \pm 2.9(7.9 ; 14)$ & $36.2 \pm 16.1(11.0 ; 13)$ & - & $<0.001$ & 0.004 & 1.00 & $<0.001$ \\
\hline
\end{tabular}

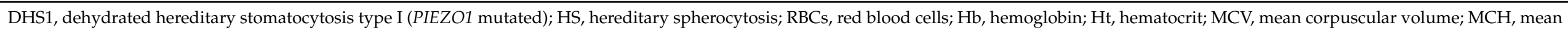

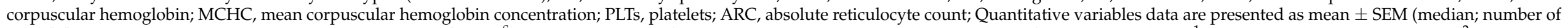

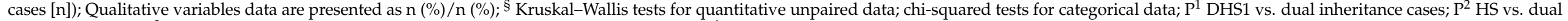
inheritance cases; $\mathrm{P}^{3}$ DHS1 vs. HS cases (Bonferroni post-hoc tests for multiple comparisons); ${ }^{+}$Normalization of ferritin using "Ferritin level/dosage age ratio", as described by [16]. 


\subsection{Hydration and Deformability Status of Dual Inheritance Patients}

To investigate the multi-locus contributions to the hydration and deformability of RBCs of some representative dual inheritance patients $(n=11)$, the ektacytometry curves were analyzed in comparison with DHS1 $(n=18$, patients with clinical and molecular diagnosis described in [16]) and HS ( $n=116$, on the basis of the recently published study [17]). Some of these Osmoscan profiles for the dual inheritance patients are reported in Figure S3. Three Osmoscan parameters were evaluated across the three subgroups of patients: (i) the minimum osmolality of ' $\mathrm{O}$ min', as the osmolality at which the deformability reaches a minimum, which represents $50 \%$ of RBC hemolysis in conventional osmotic fragility assays, and reflects the mean cell surface:volume ratio; (ii) the maximum elongation index as 'EI max', which corresponds to the maximal deformability or elongation obtained near the isotonic osmolality, and is an expression of the membrane surface; and (iii) the hypertonic osmolality as 'O hyper', which represents the osmolality in the hypertonic region that corresponds to $50 \%$ of the EI max, and which reflects the mean cell hydration status. Overall, there were no significant differences in 'Omin' and 'EI max' between dual inheritance and DHS1 (Figure 2B). Indeed, these analyses demonstrated lower O min and O hyper for dual inheritance compared to HS, while EI max was significantly higher in dual inheritance compared to HS (Figure 2B). The parameter 'O hyper' was decreased in dual inheritance compared to both DHS1 and HS demonstrating a substantial dehydration status of RBCs (Figure 2B).

\section{Discussion}

Multi-locus inheritance defines a genetic disease that arises from mutations to more than one gene. Such multi-locus cases can be caused by (i) biallelic or triallelic mutations in two distinct genes, in cis or trans; (ii) co-inheritance of pathogenic variants responsible for two or more distinct disease entities, which can lead to a mixed phenotype; or (iii) pseudo-multi-locus inheritance due to monogenic Mendelian conditions, with a broad spectrum of phenotypes due to co-inheritance of genetic modifiers [18]. It is now clear that many Mendelian conditions can be characterized by complex modes of inheritance. In this context, NGS-based genetic testing has revolutionized the diagnosis of genetic diseases by identification of multi-locus inheritance for several diseases, such as Charcot-Marie-Tooth disease [19], polycystic kidney disease [20], and congenital hypogonadotropic hypogonadism [21].

In the present study, we examined multi-locus inheritance in patients with RBC defects. A case series of 155 consecutive patients were examined who were referred in the Medical Genetics Unit ('Federico II' University Hospital, Naples, Italy) from January 2018 to September 2020. The genetic analysis was conducted using a third version of a custom targeted NGS panel that included 86 causative genes of hereditary RBC defects. We obtained an overall diagnostic yield of $84 \%$ of the patients, thus improving the diagnostic yield previously reached by the first ( 34 genes) and second ( 71 genes) versions of these NGS panels targeted for RBC defects $(65 \%)[13,22]$. This is in agreement with diagnostic rates reported in the literature. Indeed, the custom panels for hereditary anemias that are available include variable numbers of genes (e.g., 50-200) with diagnostic rates reported from $38 \%$ to $87 \%$, which depend on how many and which genes are included, and on the depth of the phenotypic assessment required [9]. Our diagnostic workflow provides the use of whole exome sequencing in the negative cases to find new causative genes of RBC defects, or of CGH-array to find possible deletion/duplication.

In the present cohort of patients, $69 \%$ showed monogenic inheritance and $15 \%$ multilocus inheritance, as mainly a dual molecular diagnosis. These data are in agreement with the literature. Indeed, dual molecular diagnoses have been reported to account for at least $4 \%$ of diagnosed cases [23], with higher rates seen for case series of selected phenotypes $(12 \%)$ [24] and for cases with apparent phenotypic expansion (32\%) [25].

Here, most of these dual inheritance cases were due to co-inheritance of pathogenic variants of the PIEZO1 and SPTA1 genes, which are responsible for two distinct diseases: 
DHS1 and HS. Accordingly, these genes were the most frequently mutated loci among the causative genes identified in the present case series. PIEZO1 and SPTA1 are two large and highly polymorphic genes that show reduced genetic constraints. Of note, our data suggested that ACMG classification fails to assess the pathogenicity of genetic variants in both genes. Indeed, although the ACMG guidelines were intended to be used universally for all Mendelian disorders, certain criteria require gene- or disease-specific knowledge for an accurate variant interpretation [26]. The mutated genes identified here were classified based on their genic intolerance, using RVIS percentile scores. According to their low intolerance to variation, the PIEZO1 and SPTA1 genes showed the highest RVIS values. Indeed, the more intolerant to variation a gene is, the less likely it is to be mutated. Interestingly, among the causative genes described here, PKLR and SEC23B also showed high frequencies of mutations, and accordingly, they showed intermediate RVIS percentiles. This might explain the high prevalence of patients with congenital dyserythropoietic anemia II among those with congenital dyserythropoietic anemias, as well as the increased number of patients diagnosed with pyruvate kinase deficiency in recent years [3].

The multi-locus inheritance led to a mixed phenotype that was more similar to HS than DHS1, in terms of the RBC indices of $\mathrm{Hb}, \mathrm{MCV}$, and MCH. Interestingly, as assessed by the ferritin:age ratio, the iron balance of these dual inheritance cases was also more similar to HS than DHS1. Therefore, in terms of the Hb levels, the clinical phenotype was more severe for these dual inheritance patients compared to DHS1. In contrast, for $\mathrm{MCV}, \mathrm{MCH}$, and the ferritin:age ratio, the phenotype of the multi-locus cases was milder compared to DHS1.

Within the present case series, there were seven severe cases who were transfusion dependent (patients P2, P3, P4, P6, P7, P10, P20). Of note, four of these seven transfusiondependent patients showed dual inheritance of DHS1/HS. The hemolytic indices for bilirubin and LDH did not differ in the multi-locus patients compared to those who were monogenic. However, most of the multi-locus patients had splenomegaly, and four of them had undergone splenectomy.

Here, we also identified a rare case of a syndrome characterized by hydrops, lactic acidosis, and sideroblastic anemia, which was due to a homozygous variant in the LARS2 gene. This patient (P8) was a 3-year-old Swedish female who showed multi-locus inheritance of variants in the $A B C B 6, K C N N 4$, and LARS2 genes. Indeed, she had microcytic anemia with fetal ascites that required two intrauterine transfusions. She also showed impairment of psychomotor development and bilateral deafness.

We further characterized the deformability and hydration status of RBCs from the patients with dual inheritance by ektacytometry analysis. Here, the co-inheritance of DHS1 and HS resulted in peculiar bell-shaped ektacytometry curves that were left shifted, as for the patients with DHS1. Indeed, comparisons of the 'O hyper' parameter (which reflects the hydration status) among these dual inheritances, DHS1, and HS patients highlighted lower values for dual inheritance compared to both DHS1 and HS. Overall, comparisons of these ektacytometry curves for dual inheritance with those of DHS1 and HS highlighted the dehydrated conditions of the RBCs, as seen for DHS1. The 'O min' value, which reflects the mean cell surface:volume ratio, was more similar to DHS1. Finally, the 'EI max' (as an expression of the cell membrane surface) was higher for the multi-locus cases compared to both DHS1 and HS.

\section{Conclusions}

In this study, we evaluated a large case series of 155 consecutive patients with different forms of RBC defects who were referred to the Medical Genetics Unit ('Federico II' University Hospital, Naples, Italy) for NGS-based genetic testing, from January 2018 to September 2020. Among the diagnosed patients, $15 \%$ showed multi-locus inheritance, which mainly involved PIEZO1 and SPTA1 variants. The data from the present study demonstrate that the first and second line of investigations included in the conventional workflow for diagnosis of hereditary anemias can fail to provide differential diagnoses for patients with 
multi-locus inheritance. Indeed, we have shown that the clinical parameters, such as the $\mathrm{RBC}$ indices and the iron status, are not informative for any differential diagnosis of dual inherited conditions. Moreover, the Osmoscan profile did not provide easy discrimination between these multi-locus and monogenic RBC defects.

Of note, our study further highlighted the importance to reevaluate the pathogenicity of the identified genetic variants in light of the new data presented in the literature and of the follow-up of the clinical case. For PIEZO1 and SPTA1 genes, we demonstrated that the ACMG rules often failed to assess the pathogenicity of the identified variants. For this reason, the introduction of functional tests is useful to define the pathogenicity of VUS and to establish a correct genotype-phenotype relationship.

Genetic testing is already a routine part of the diagnostic workflow for patients with RBC defects, and is indeed widespread in clinical practice. Additionally, genetic testing is used more early in the diagnostic workflow of hereditary anemias [8,9]. These data further demonstrate the crucial role for NGS-based genetic testing for diagnosis of such RBC defects, and also for the identification of multi-locus inheritance. Correct genetic diagnosis has become important also to guide treatment and personalized clinical management of these patients. For example, for patients with multi-locus inheritance caused by DHS1 and HS, it is crucial to avoid splenectomy, which is beneficial for patients with HS but contraindicated for DHS1.

Supplementary Materials: The following are available online at https:/ /www.mdpi.com/article/ 10.3390/genes12070958/s1, Figure S1: Histogram chart showing the ACMG classification of the PIEZO1 and SPTA1 known causative variants. Figure S2: Pedigree of nine probands with digenic inheritance. Figure S3. Osmoscan profiles of some representative dual inheritance cases. Table S1. Additional evidence supporting variant reassessment. Table S2. Clinical characteristics of the patients with multi-locus inheritance.

Author Contributions: I.A. and R.R. designed and conducted the study, and prepared the manuscript; S.M. performed clinical evaluation of the patients, and collected clinical data; R.R. and I.A. performed in-silico design of the NGS panels, and interpretation of the genetic variants; B.E.R. and R.M. performed library enrichment and Sanger sequencing analysis; S.M., A.G., G.L.F., V.P. (Valeria Pinto), M.G. (Magnus Göransson), V.P. (Vasiliki Papadopoulou), M.G. (Mathilde Gavillet), M.E. provided samples and cared for the patients; A.P. and G.T. performed the ektacytometry analysis; A.I. carried out critical revision of the manuscript. All authors have read and agreed to the published version of the manuscript.

Funding: This research was funded by an EHA Junior Research Grant to Immacolata Andolfo (3978026), and by Bando Star Linea 1-Junior Principal Investigator Grants-COINOR, Università degli Studi di Napoli 'Federico II' to Roberta Russo.

Institutional Review Board Statement: The local University Ethical Committee approved the collection of the patient data (DAIMedLab, 'Federico II' University of Naples; $\mathrm{N}^{\circ} 252 / 18$ ).

Informed Consent Statement: DNA samples were obtained from the patients after they had signed their informed consent, and according to the Declaration of Helsinki.

Conflicts of Interest: The authors declare no conflict of interest.

\section{References}

1. Da Costa, L.; Narla, A.; Mohandas, N. An update on the pathogenesis and diagnosis of Diamond-Blackfan anemia. F1000Research 2018, 7. [CrossRef]

2. Iolascon, A.; Andolfo, I.; Russo, R. Advances in understanding the pathogenesis of red cell membrane disorders. Br. J. Haematol. 2019, 187, 13-24. [CrossRef]

3. Iolascon, A.; Andolfo, I.; Russo, R. Congenital dyserythropoietic anemias. Blood 2020, 136, 1274-1283. [CrossRef] [PubMed]

4. Andolfo, I.; Russo, R.; Gambale, A.; Iolascon, A. Hereditary stomatocytosis: An underdiagnosed condition. Am. J. Hematol. 2018, 93, 107-121. [CrossRef]

5. Andolfo, I.; Russo, R.; Gambale, A.; Iolascon, A. New insights on hereditary erythrocyte membrane defects. Haematologica 2016, 101, 1284-1294. [CrossRef] [PubMed]

6. Taher, A.T.; Weatherall, D.J.; Cappellini, M.D. Thalassaemia. Lancet 2018, 391, 155-167. [CrossRef] 
7. Bianchi, P.; Fermo, E.; Lezon-Geyda, K.; van Beers, E.J.; Morton, H.D.; Barcellini, W.; Glader, B.; Chonat, S.; Ravindranath, Y.; Newburger, P.E.; et al. Genotype-phenotype correlation and molecular heterogeneity in pyruvate kinase deficiency. Am. $J$. Hematol. 2020, 95, 472-482. [CrossRef]

8. Roy, N.B.A.; Babbs, C. The pathogenesis, diagnosis and management of congenital dyserythropoietic anaemia type I. Br. J. Haematol. 2019, 185, 436-449. [CrossRef]

9. Russo, R.; Marra, R.; Rosato, B.E.; Iolascon, A.; Andolfo, I. Genetics and Genomics Approaches for Diagnosis and Research Into Hereditary Anemias. Front. Physiol. 2020, 11, 613559. [CrossRef]

10. Posey, J.E. Genome sequencing and implications for rare disorders. Orphanet J. Rare Dis. 2019, 14, 153. [CrossRef]

11. Russo, R.; Langella, C.; Esposito, M.R.; Gambale, A.; Vitiello, F.; Vallefuoco, F.; Ek, T.; Yang, E.; Iolascon, A. Hypomorphic mutations of SEC23B gene account for mild phenotypes of congenital dyserythropoietic anemia type II. Blood Cells Mol. Dis. 2013, 51, 17-21. [CrossRef] [PubMed]

12. Andolfo, I.; Martone, S.; Ribersani, M.; Bianchi, S.; Manna, F.; Genesio, R.; Gambale, A.; Pignataro, P.; Testi, A.M.; Iolascon, A.; et al. Apparent recessive inheritance of sideroblastic anemia type 2 due to uniparental isodisomy at the SLC25A38 locus. Haematologica 2020, 105, 2883-2886. [CrossRef]

13. Russo, R.; Andolfo, I.; Manna, F.; Gambale, A.; Marra, R.; Rosato, B.E.; Caforio, P.; Pinto, V.; Pignataro, P.; Radhakrishnan, K.; et al. Multi-gene panel testing improves diagnosis and management of patients with hereditary anemias. Am. J. Hematol. 2018, 93, 672-682. [CrossRef] [PubMed]

14. Richards, S.; Aziz, N.; Bale, S.; Bick, D.; Das, S.; Gastier-Foster, J.; Grody, W.W.; Hegde, M.; Lyon, E.; Spector, E.; et al. Standards and guidelines for the interpretation of sequence variants: A joint consensus recommendation of the American College of Medical Genetics and Genomics and the Association for Molecular Pathology. Genet. Med. 2015, 17, 405-424. [CrossRef]

15. Petrovski, S.; Wang, Q.; Heinzen, E.L.; Allen, A.S.; Goldstein, D.B. Genic intolerance to functional variation and the interpretation of personal genomes. PLoS Genet. 2013, 9, e1003709. [CrossRef]

16. Andolfo, I.; Russo, R.; Rosato, B.E.; Manna, F.; Gambale, A.; Brugnara, C.; Iolascon, A. Genotype-phenotype correlation and risk stratification in a cohort of 123 hereditary stomatocytosis patients. Am. J. Hematol. 2018, 93, 1509-1517. [CrossRef]

17. Huisjes, R.; Makhro, A.; Llaudet-Planas, E.; Hertz, L.; Petkova-Kirova, P.; Verhagen, L.P.; Pignatelli, S.; Rab, M.A.; Schiffelers, R.M.; Seiler, E.; et al. Density, heterogeneity and deformability of red cells as markers of clinical severity in hereditary spherocytosis. Haematologica 2020, 105, 338-347. [CrossRef]

18. Deltas, C. Digenic inheritance and genetic modifiers. Clin. Genet. 2018, 93, 429-438. [CrossRef]

19. Barreda Fierro, R.; Herrera Mora, P.; Zenteno, J.C.; Villarroel Cortes, C.E. Clinical and molecular evidence of possible digenic inheritance for MFN2/GDAP1 genes in Charcot-Marie-Tooth disease. Neuromuscul. Disord. 2020, 30, 986-990. [CrossRef]

20. Durkie, M.; Chong, J.; Valluru, M.K.; Harris, P.C.; Ong, A.C.M. Biallelic inheritance of hypomorphic PKD1 variants is highly prevalent in very early onset polycystic kidney disease. Genet. Med. 2021, 23, 689-697. [CrossRef]

21. Neocleous, V.; Fanis, P.; Toumba, M.; Tanteles, G.A.; Schiza, M.; Cinarli, F.; Nicolaides, N.C.; Oulas, A.; Spyrou, G.M.; Mantzoros, C.S.; et al. GnRH Deficient Patients with Congenital Hypogonadotropic Hypogonadism: Novel Genetic Findings in ANOS1, RNF216, WDR11, FGFR1, CHD7, and POLR3A Genes in a Case Series and Review of the Literature. Front. Endocrinol. 2020, 11, 626. [CrossRef] [PubMed]

22. Al-Riyami, A.Z.; Iolascon, A.; Al-Zadjali, S.; Andolfo, I.; Al-Mammari, S.; Manna, F.; Al Rawas, A.; King, M.J.; Russo, R. Targeted next generation sequencing identifies a novel $\beta$-spectrin gene mutation A2059P in two Omani children with hereditary pyropoikilocytosis. Am. J. Hematol. 2017, 92, E607-E609. [CrossRef] [PubMed]

23. Posey, J.E.; Harel, T.; Liu, P.; Rosenfeld, J.A.; James, R.A.; Coban Akdemir, Z.H.; Walkiewicz, M.; Bi, W.; Xiao, R.; Ding, Y.; et al. Resolution of Disease Phenotypes Resulting from Multilocus Genomic Variation. N. Engl. J. Med. 2017, 376, 21-31. [CrossRef] [PubMed]

24. Tarailo-Graovac, M.; Shyr, C.; Ross, C.J.; Horvath, G.A.; Salvarinova, R.; Ye, X.C.; Zhang, L.H.; Bhavsar, A.P.; Lee, J.J.; Drögemöller, B.I.; et al. Exome Sequencing and the Management of Neurometabolic Disorders. N. Engl. J. Med. 2016, 374, 2246-2255. [CrossRef]

25. Karaca, E.; Posey, J.E.; Akdemir, Z.C.; Pehlivan, D.; Harel, T.; Jhangiani, S.N.; Bayram, Y.; Song, X.; Bahrambeigi, V.; Yuregir, O.O.; et al. Phenotypic expansion illuminates multilocus pathogenic variation. Genet. Med. 2018, 20, 1528-1537. [CrossRef]

26. Pepin, M.G.; Murray, M.L.; Bailey, S.; Leistritz-Kessler, D.; Schwarze, U.; Byers, P.H. The challenge of comprehensive and consistent sequence variant interpretation between clinical laboratories. Genet. Med. 2016, 18, 20-24. [CrossRef] 